EESTI NSV TEADUSTE AKADEEMIA TOIMETISED. 25. KOIDE

FOUSIKA * MATEMAATIKA. 1976, NR. 4

ИЗВЕСТИЯ АКАДЕМИИ НАУК ЭСТОНСКОИ ССР. ТОМ 25 ФИЗИКА * МАТЕМАТИКА, 1976, № 4

\title{
К ОПРЕДЕЛЕНИЮ ТЕМПЕРАТУРНЫХ НАПРЯЖЕНИЙ В ЦИЛИНДРАХ МЕТОДОМ ИНТЕГРАЛЬНОЙ ФОТОУПРУГОСТИ
}

E. BROSMAN. TEMPERATUURIPINGETE MÄARAMINE SILINDRITES INTEGRAALSEL FOTÒELASTSUSMEETODIL

E. BROSMAN. DETERMINATION OF THERMAL STRESSES IN CYLINDERS BY INTEGRATED PHOTOELASTICITY

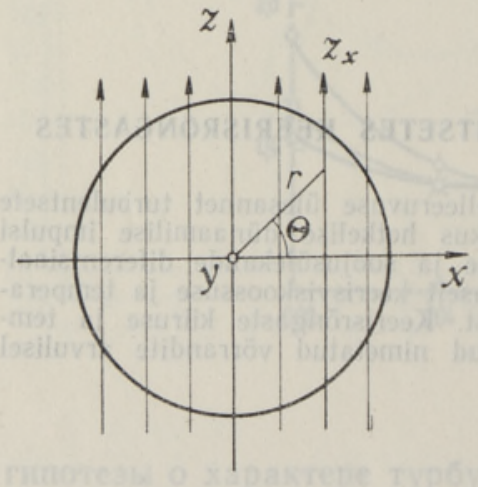

Для определения остаточных температурных напряжений в прозрачных цилиндpax кругового поперечного сечения $x z$ (рис. 1) при отсутствии градиента напряжений в направлении оси цилиндра необходимо знать распределение интегральной разности хода $\delta(x)$. Поскольку нет вращения квазиглавных направлений на пути света, имеет место интегральный закон Вертгейма, что дает для луча света, находящегося на расстоянии $x$ от оси $z$ :

Рис. 1. Схема просвечивания кругового цилиндра.

$$
\delta(x)=2 C \int_{0}^{z_{x}}\left[\sigma_{y}(r)-\sigma_{r}(r) \cos ^{2} \Theta-\sigma_{\Theta}(r) \sin ^{2} \Theta\right] d z,
$$

где $C$ - упругооптический коэффициент.

Из условия равновесия сегмента поперечного слоя цилиндра вытекает $\left[{ }^{1}\right]$

$$
\int_{0}^{z_{x}}\left(\sigma_{r} \cos ^{2} \Theta+\sigma_{\Theta} \sin ^{2} \Theta\right) d z=0
$$

Следовательно, разность хода обусловливается лишь продольным напряжением $\sigma_{y}$ :

$$
\delta(x)=2 C \int_{0}^{z_{x}} \sigma_{y} d z .
$$

Выражая $\sigma_{y}$ в виде степенного ряда [2]

$$
\sigma_{y}=\sum_{k=0}^{n} a_{2 k} r^{2 k}, \quad 1 \geqslant r \geqslant 0,
$$


получаем после интегрирования выражения (3) линейную систему уравнений

$\delta\left(x_{i}\right) / 2 C R=\sum_{k=0}^{n} a_{2 k} G_{2 k}\left(x_{i}\right), \quad 1 \geqslant x_{i} \geqslant-1, \quad i=1,2, \ldots, m ; m>n$,

где $R$ - радиус цилиндра,

$$
G_{2 k}\left(x_{i}\right)=\sum_{l-1,3,5 \ldots}^{2 k+1}\left(1-x_{i}^{2}\right)^{l / 2} x_{i}^{2 k+1-l} C_{2 k+1}^{l} / l .
$$

В уравнении (6) $C_{2 k+1}^{l}-$ биномиальные коэффициенты Ньютона. Из системы (5) можно вычислить коэффициенты $a_{2 k}$, которые и определят распределение $\sigma_{y}$ согласно соотношению (4).

Полученную систему линейных уравнений (5) можно решить методом наименьших квадратов. В зависимости от условий возникновения напряжений имеет место т. н. закон суммы [3]

$$
k \sigma_{y}=\sigma_{r}+\sigma_{\Theta}
$$

где $k=1$ или $k=\mu$ ( $\mu-$ коэффициент Пуассона). Подстановка соотношения (7) в уравнение равновесия

$$
d\left(r \sigma_{r}\right) / d r-\sigma_{\Theta}=0
$$

приводит к линейному дифференциальному уравнению

$$
d \sigma_{r} / d r+2 \sigma_{r} / r=k \sigma_{y} / r
$$

решение которого, с учетом разложения (4), можно выразить в виде

$$
\sigma_{r}(r)=k \sum_{k=0}^{n} a_{2 k} r^{2 k} /(2 k+2) .
$$

Окончательное решение задачи принимает вид

$$
\begin{aligned}
& \sigma_{y}(r)=\sum_{k=0}^{n} a_{2 k} r^{2 k}, \\
& \sigma_{r}(r)=k \sum_{k=0}^{n} a_{2 k} r^{2 k} /(2 k+2), \\
& \sigma_{\Theta}(r)=k \sum_{k=0}^{n} a_{2 k} r^{2 k}(2 k+1) /(2 k+2) .
\end{aligned}
$$

Полученный результат отличается от известного из литературы результата $\left[{ }^{4}\right]$ тем, что компоненты напряжения описываются в элементарных функциях и на оси цилиндра компоненты напряжений $\sigma_{r}$ и $\sigma_{\theta}$ определяются точно.

В качестве примера описанная выше методика была использована для определения напряжения в закаленном цилиндре из стекла марки № 29. Измерения разностей хода проводились в иммерсионной ванне на полярископе КСП-5 с помощью компенсатора СКК-2. Радиус цилиндра $R=1,2 c M$, упругооптический коэффициент $C=2,0 \cdot 10^{-7} C M^{2} / \kappa \Gamma$, коэффициент $k=1$.

График измеренного распределения разности хода показан на рис. 2. Эксперимент подтверждает теоретический вывод $\left[{ }^{4}\right]$

$$
\int_{-R}^{R} \delta(x) d x=0 .
$$




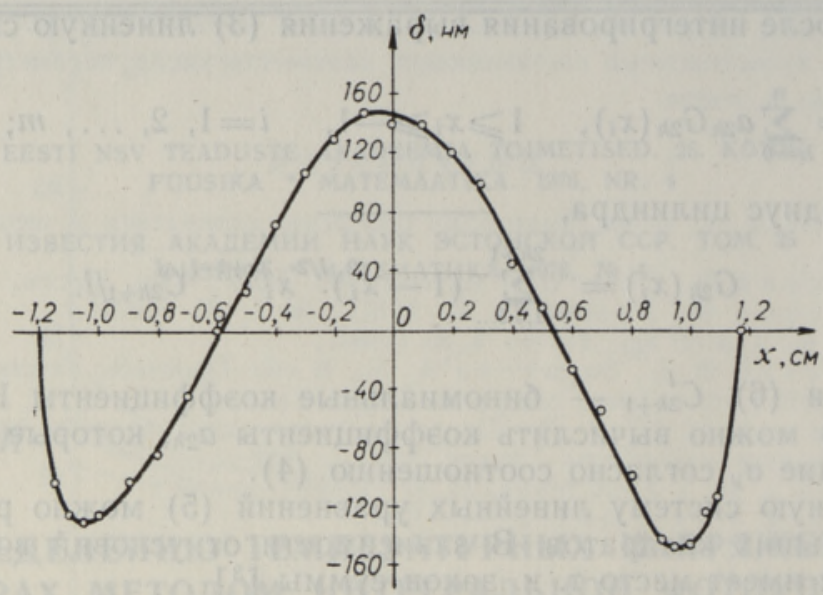

Рис. 2. Распределение интегральной разности хода в цилиндре.

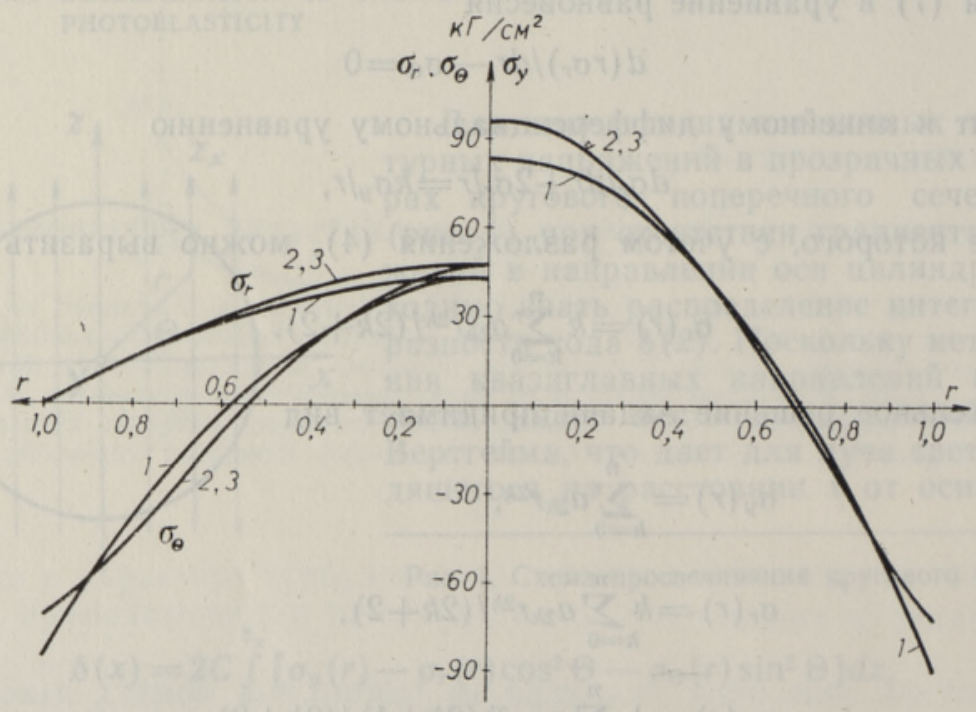

Рис. 3. Распределение напряжений $\sigma_{y}, \sigma_{r}$ и $\sigma_{\Theta}$ при разных степенях приближения (13).

Решение задачи на ЭВМ «Минск-32» подтвердило также предсказания теории $\left[{ }^{5}\right]$ о том, что распределение продольных напряжений близко к параболическому. В различных приближениях были получены следующие результаты:

$$
\begin{aligned}
& \sigma_{y}=85,7-171,6 r^{2}, \\
& \sigma_{y}=93,6-198,0 r^{2}+78,0 r^{4}, \\
& \sigma_{y}=93,5-241,8 r^{2}+65,0 r^{4}+8,0 r^{6} .
\end{aligned}
$$

Графически распределение компонентов напряжений $\sigma_{y}, \sigma_{r}$ и $\sigma_{\theta}$ изображено на рис. 3. Видно, что на краю цилиндра распределение напряжений несколько отличается от параболического. Такая же картина на- 
блюдалась при исследовании остаточных напряжений, возникающих в пластинках при их охлаждении от высоких температур [6].

Среднее значение напряжения $\sigma_{y}$ по всему поперечному сечению $\int_{0}^{1} r \sigma_{y}(r) d r=1,6 \quad \kappa \Gamma / c м^{2}$ составляет $1,7 \%$ от его максимального значения. Следовательно, это распределение можно считать практически уравновешенным.

\section{ЛИТ Е Р А Т Р А}

1. Poritzky H., Phys., 5, No. 12, 406 (1934).

2. А бен Х. К., Интегральная фотоупругость, Таллин, 1975.

3. Инденбом В. Л., Житомирский И. С., Чебанова Г. С., Кристаллография, 18, № 1, 39 (1973).

4. O'Rourke R. C., S a e n z A. W., Quart. Appl. Math., 8, No. 3, 303 (1950).

5. Sutt on P. M., J. Amer. Ceram. Soc., 41, No. 3, 103 (1958).

6. Резников Б. А., ФТТ, 5, № 9, 2526 (1963).

Ннститут кибернетики Академии наук Эстонской ССР
Поступила в редакцию

$5 / \mathrm{I} \quad 1976$ 